\title{
A multi-disciplinary approach to improving outcomes for patients receiving parenteral nutrition in a District General Hospital
}

\author{
C. Patnaik, D. Spalding, K. Jones, K. Laland, K. R. Banfield and K. G. Kerr \\ Harrogate and District Foundation Trust, Lancaster Park Road, Harrogate HG2 7SX,UK
}

In early 2006, a small cluster of eight patients developed parenteral nutrition (PN) line related infections ( $n=7$ Candidaemia; $n=1$ Bacteraemia) identified by positive blood cultures at Harrogate and District Foundation Trust. PN guidelines had been developed in 2003; however, these were not approved through any formal process (Clinical governance) and many staff were unaware of their existence. This led to the formation of an action team consisting of representatives from Pharmacy, Nutrition and Dietetics and Infection Control. Initial analysis of the existing PN policy highlighted the following issues. The number of patients receiving PN was approximately 1-2 per month with many patients on PN for less than 5 days; the decision to start PN in individual patients was without prior discussion with a Dietician or Pharmacist. It was therefore very likely that many patients may have received PN without assessing suitability for enteral nutrition first. Investigations conducted by Infection control team suggested that PN-related line infections may have been due to contamination of venous catheters most likely a result of sub-optimal catheter care.

As a result of the above, the PN policy and guidelines were re-written and accepted by Trust's Governance process in January 2007. The Infection Control Policy for intravenous procedures was revised and re-issued. The prescribing of PN was restricted to those patients who have had a multidisciplinary assessment (patient's consultant, pharmacist and dietician). The prescribing of PN was restricted outside of normal working hours and at weekends where it would be difficult to conduct a multidisciplinary assessment. Unless there were compelling reasons to the contrary, patients requiring PN must have a single-lumen line inserted for the purpose and subsequent study days for senior nurses on aspects of intravenous line care were held in February 2007. The Infection control team monitors patients receiving PN closely.

\begin{tabular}{lrrrr}
\hline Year & 2006 & 2007 & 2008 & 2009 \\
\hline Number of patients prescribed PN & 27 & 17 & 15 & 14 \\
Number of patients prescribed PN for less than 5 days & 10 & 5 & 6 & 6 \\
Number of patients with PN related line infections & 8 & 1 & 0 & 0 \\
Incidence rate of infection per 1000 catheter days & 17 & 5 & 0 & 0 \\
\hline
\end{tabular}

Since implementation of the PN policy, all new requests for PN are discussed with the requesting consultant, dietician and pharmacist. Appropriate prescribing of PN has resulted in fewer patients receiving PN. There has been one case of PN related line infection since the implementation of the PN policy. The results of this simple intervention clearly demonstrate the impact of a multidisciplinary approach to improving outcomes for patients receiving PN. 